\title{
Self-limiting evolution of seeded quantum wires and dots on patterned substrates
}

\author{
V. Dimastrodonato, ${ }^{1}$ E. Pelucchi, ${ }^{1}$ and D. D. Vvedensky ${ }^{2}$ \\ ${ }^{1}$ Tyndall National Institute, University College Cork, \\ "Lee Maltings," Dyke Parade, Cork, Ireland, and \\ ${ }^{2}$ The Blackett Laboratory, Imperial College London, London SW7 2AZ, United Kingdom
}

(Dated: April 11, 2015)

\begin{abstract}
Extensive experimental data and an accompanying theoretical model are presented for the selflimiting profiles and Ga segregation on patterned GaAs(111)B substrates during metalorganic vaporphase epitaxy of $\mathrm{Al}_{x} \mathrm{Ga}_{1-x}$ As. Self-limiting widths and segregation of Ga produce quantum dots along the base of pyramidal recesses bounded by (111)A planes and quantum wires along the vertical axis of the template, respectively. Coupled reaction-diffusion equations for precursor and adatom kinetics reproduce the measured concentration- and temperature-dependence of the self-limiting width and segregation. Our model can be extended to other patterned systems, providing a new paradigm for predicting the morphology of surface nanostructures and inferring their quantum optical properties.
\end{abstract}

PACS numbers: 68.55.-a, 68.65.-k, 81.05.Ea, 81.10.Aj, 81.10.Bk

The morphological evolution of a patterned crystalline surface driven by an incident flux of matter is among the most intriguing phenomena of surface physics. The main characteristics of growth morphologies on such substrates are due to different exposed facets having different kinetic and chemical properties. Processes such as the migration of precursors and adatoms, the dissociation of precursors, and the incorporation of adatoms each occur at facet-dependent rates. This results in a nonuniform growth rate across the substrate [1,2], with adjacent regions having different thicknesses and compositions which, for semiconductors, produces spatiallyvarying band gaps and, hence, carrier confinement [2]. Etched patterns can be used to channel mobile surface species to pre-determined regions of a substrate, yielding uniform arrays of low-dimensional nanostructures [2-9].

The foregoing scenario is the basis for forming quantum wires (QWRs) at V-grooves $[2,5]$ and quantum dots (QDs) at inverted pyramids [9] during metalorganic vapor-phase epitaxy (MOVPE) on etched GaAs substrates. These nanostructures are called "self-limiting" [10] because they result from the balance between the greater inherent growth rate of the side facets and capillarity, which favors growth on the bottom facet. Hence, the structural and compositional profiles of QWRs and QDs depend only on the bounding facets of the original pattern, the growth conditions, and alloy composition. This ensures reproducible lateral confinement and precise positioning, enabling the flexible and versatile design of optical nanosystems through the direct connection between geometry and excitonic spectra (see below).

Pyramidal QDs have wide-ranging properties and applications including, (i) the reproducible formation of excitonic states, which provides uniform single photon emission [11-13], (ii) precise coupling in quantum electrodynamic cavities [14] because of accurate site- and energy-control, and (iii) efficient emission of polarizationentangled photons [15], owing to the high-symmetry [111] growth orientation and the spatial uniformity provided by the patterned substrate. The dependence of the optical transition polarization on the QD shape, size and symmetry [16] has recently been extended to an analysis that reveals the delicate interplay between the excitonic fine structure and the symmetry and composition of QDs [17]. Identifying the atomistic mechanisms responsible for the self-limiting profile would enable direct control over the geometry of the nanostructure. This would lead to reduced fine-structure effects, pave the way toward more reliable sources of entangled photons, and have an immediate and far-reaching impact on the field of quantum information.

Yet, despite the evident broad appeal of pyramidal QDs, their atomistic formation mechanisms are poorly understood. The systematic characterization of the selflimiting profile as a function of the growth conditions and composition, a key ingredient in model development, has not been readily available because of the painstaking procedures needed to acquire these data. On the other hand, previous work [18] has indicated that a reactiondiffusion model based on the surface diffusion and decomposition at step edges of the group-III precursor explains the morphology of misoriented GaAs(001) surfaces during MOVPE. In fact, the large length scales of typical etched patterns effectively pre-empt kinetic Monte Carlo simulations, except for qualitative studies [19], leaving a continuum description as the only viable alternative for a quantitative theory.

We present here extensive experimental data and an accompanying theoretical model of self-limiting growth by MOVPE of AlGaAs QDs within tetrahedral recesses patterned on GaAs(111)B surfaces. Our study provides experimental evidence for the self-limiting profile as a function of both alloy content and growth temperature $\left(T_{G}\right)$, and explains the experimental trends in terms of reaction-diffusion equations for the main steps of the growth process. Two sets of AlGaAs/GaAs samples were 

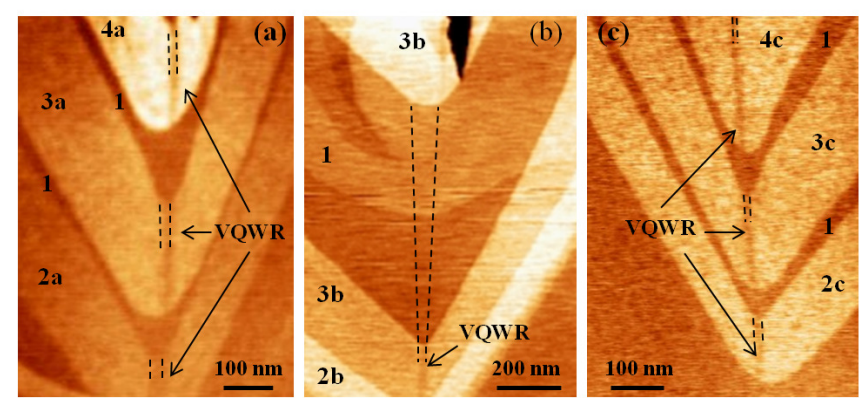

FIG. 1. (Color online) Representative flattened [23] AFM cross-sectional scans of pyramidal systems consisting of pairs of $\mathrm{Al}_{x} \mathrm{Ga}_{1-x} \mathrm{As}$ (brighter) and GaAs markers (darker, indicated with 1 ), grown by varying the alloy composition (batch I, (a) and (b)) and the growth temperature (batch II, (c)). (a) $x=0.2,0.4,0.65$ (layers $2 \mathrm{a}, 3 \mathrm{a}$ and $4 \mathrm{a}$, respectively) and (b) $x=0.75,0.55,0$ (layers $2 \mathrm{~b}, 3 \mathrm{~b}$, and 1 , respectively) to reproduce the modulation of the profile. (c) The widening of the $\mathrm{Al}_{0.3} \mathrm{Ga}_{0.7} \mathrm{As}$ self-limited profile is reproduced by increasing the temperature from $\sim 881 \mathrm{~K}$, to $904 \mathrm{~K}$, and then to $926 \mathrm{~K}$ (layer 4c, 3c and 2c respectively). Vertical QWRs (VQWRs) self-form along the vertical axis of the structure during the deposition of AlGaAs due to $\mathrm{Ga}$ segregation. The cleavage plane intersects the vertex and midpoint of the opposite base of the triangular recess, resulting in different apparent thickness on the left and right sides of the central axis.

grown with the goal of reproducing (I) the modulation of the $\mathrm{Al}$ content of the ternary compound and (II) the variation of $T_{G}$ (at a fixed alloy composition) [20]. All samples were structurally characterized by atomic force microscopy (AFM) with systematic profiling analyses, involving measurement and image processing routines, and minimizing any geometrical and AFM tip artefacts [21]. Our results establish a new paradigm for the future development of seeded nanostructures based on the judicious combination of experimental measurements and theoretical modelling, which could play a pivotal role when a particular design is needed for specific requirements.

Figures 1(a,b) show AFM cross-sectional scans of two representative samples in batch I. Figure 1(a) tracks the evolution of the self-limiting profile for several $\mathrm{Al}$ compositions. An increase of $\mathrm{Al}$ content causes a narrowing of the base profile width along the vertical axis of the template (the dashed black lines, drawn only on the upper part of each layer, are a guide to the width narrowing). This effect is especially apparent when pure GaAs is grown on $\mathrm{Al}_{0.55} \mathrm{Ga}_{0.45} \mathrm{As}$, where a sharpening of the profile is clearly evident [highlighted by the black dashed line in Fig. 1(b)]. Figure 1(c) shows how the self-limiting profile changes with $T_{G}$ at constant $\mathrm{Al}$ content. A systematic analysis of the samples from batch II reveals that increasing $T_{G}$ enhances the capillarity [22] from the sidewalls to the bottom, which increases the growth rate on the base and, therefore, broadens the basal profile.

The self-limited width of the QDs in the inverted pyra-

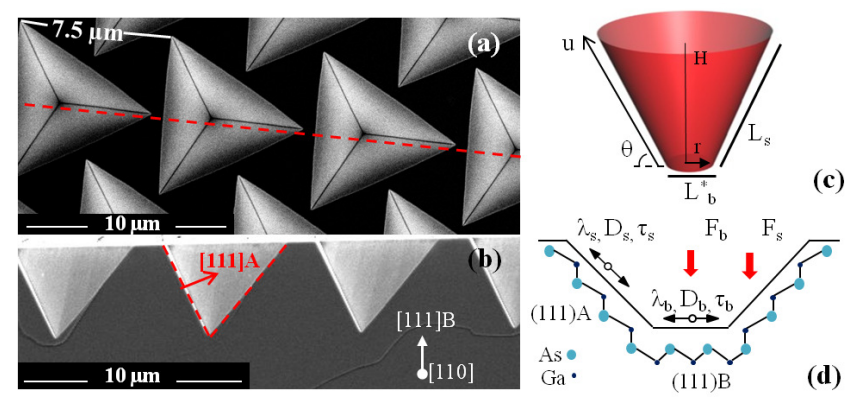

FIG. 2. (Color online) Representative scanning electron micrographs in top (a) and cross-sectional (b) view of GaAs(111)B patterned with inverted tetrahedral pyramids with a pitch of $7.5 \mu \mathrm{m}$. The lateral facet edge is highlighted in red and its [111] A crystallographic orientation is indicated in (b). (c) Truncated conical model of a pyramidal recess. (d) Cross-section of the recess along the (110) plane. The parameters $F_{s, b}, \lambda_{s, b}, D_{s, b}$ and $\tau_{s, b}$ are indicated on each facet.

mids results from a combination of the geometry of the bounding facets, the kinetics on each facet, and interfacet surface diffusion. To reproduce the periodicity of the templates [Fig. 2(a)], we divide the substrate into identical unit cells, each consisting of a recess composed of a flat (111)B base and the lateral (111)A surfaces, as delivered by chemical etching after patterning [Fig. 2(b)]. Since, as we shall demonstrate, the self-limiting profile is due to the kinetics at the bottom of the recess, the top planar surface can be neglected, which effectively decouples the recesses from one another. To obtain an analytically tractable model, while retaining the essence of the pattern, we replace the pyramidal recess by an inverted truncated conical recess, as shown in Fig. 2(c). The (111)B base is a circle of diameter $L_{b}^{*}$, the length of the self-limiting profile, and the (111)A lateral facets, with length $L_{s}$, form the sides of the truncated cone.

We suppose that the precursors, trimethylgallium (TMGa), trimethylaluminum (TMAl), and arsine $\left(\mathrm{AsH}_{3}\right)$ arrive at the substrate by diffusion through a boundary layer. Because of the arsenic-rich conditions, we consider the kinetics only of the group-III species and their precursors, as the concentration of arsenic is presumed not be rate-limiting in any surface reaction [24]. Atoms are released by the decomposition of precursors at step edges, whereupon they diffuse until incorporated into the growth front. The adatom concentration $n_{i}$ on each facet $i$ is a solution of the stationary diffusion equation,

$$
D_{i} \nabla^{2} n_{i}+F_{i}-\frac{n_{i}}{\tau_{i}}=0
$$

in which $D_{i}$ is the diffusion constant, $F_{i}$ the effective atomic flux, and $\tau_{i}$ the lifetime to incorporation. There are separate equations for $\mathrm{Ga}$ and $\mathrm{Al}$ on each facet, so each quantity in this equation has a value for each atomic type [Fig. 2(d)]. We consider only stationary solutions, as our interest is the self-limiting profile, rather than the 
evolution towards this profile.

In the first term in (1), the adatom diffusion coefficient $D_{i}$ is given by the Arrhenius expression

$$
D_{i}=a^{2} \nu \exp \left(-\frac{E_{i}^{D}}{k_{B} T}\right)
$$

where $a$ is the jump length, taken as the nearest neighbour lattice spacing, $\nu \sim 10^{13} \mathrm{~s}^{-1}$ the attempt frequency, $E_{i}^{D}$ the energy barrier to hopping, $k_{B}$ is Boltzmann's constant, and $T$ the absolute temperature [25].

The effective atomic fluxes in the second term in (1) are products of precursor fluxes and their facet-dependent $[22,26]$ decomposition rates. The effective fluxes on the side and bottom regions, $F_{s}$ and $F_{b}$, respectively, are linked by $F_{s}=r F_{b}$, where $r>1$ accounts for the enhanced decomposition on the sidewalls. These fluxes are determined by requiring that the total deposition of group-III precursors corresponds to a growth rate of 1 monolayer (ML)/s and that the growth rate $F$ across the unit cell is related to the growth rates of the individual facets by

$$
A_{b} F_{b}+A_{s} F_{s}=\left(A_{b}+A_{s}\right) F,
$$

where $A_{b}$ and $A_{s}$ are the areas of the base and side facets. For $\mathrm{Al}_{x} \mathrm{Ga}_{1-x}$ As the total deposition flux of $\mathrm{Al}$ atoms is $F_{i}^{\mathrm{Al}}=x F_{i}$ while, for Ga, $F_{i}^{\mathrm{Ga}}=(1-x) F_{i}$, with $F_{i}$ calculated from (3) for each species. Once $r^{\mathrm{Al}, \mathrm{Ga}}$ are fixed, the effective atomic deposition fluxes on each facet are determined by experimentally controllable parameters.

Finally, in the third term in (1), experimental observations, mainly from molecular-beam epitaxy [27], suggest an exponentially decreasing lifetime with temperature. Hence, for the $i$ th facet, we take

$$
\frac{1}{\tau_{i}}=\nu_{i} \exp \left(-\frac{E_{i}^{\tau}}{k_{B} T}\right)
$$

with frequency prefactor $\nu_{i}$ and energy barrier $E_{i}^{\tau}$.

The concentrations of $\mathrm{Ga}$ and $\mathrm{Al}$ within the conical recess are determined from the general solutions of (1) for the bottom facet and the sidewalls. For the circular bottom facet, we express (1) in polar coordinates and obtain the (finite) solution

$$
n_{b}(r)=F_{b} \tau_{b}+C_{b} I_{0}\left(\frac{r}{\lambda_{b}}\right),
$$

with $C_{b}$ an arbitrary constant, $I_{0}$ the modified Bessel function of the first kind of order zero, and $\lambda_{b}=$ $\left(D_{b} \tau_{b}\right)^{1 / 2}$ the diffusion length on this facet.

The form of (1) on the conical side facets requires the Laplace-Beltrami operator on this curved surface [28]. The (finite) general solution of the resulting equation is

$$
n_{s}(u)=F_{s} \tau_{s}+C_{s} K_{0}\left(\frac{L_{b} / 2+u \cot \theta}{\lambda_{s} \cos \theta}\right)
$$

where $u$ is the "radial" coordinate on the cone, $\theta$ the angle between the basal and side facets [Fig. 2(c)], $C_{s}$ an arbitrary constant, $K_{0}$ the modified Bessel function of the second kind of order zero, and $\lambda_{s}=\left(D_{s} \tau_{s}\right)^{1 / 2}$ the diffusion length on this facet.

The arbitrary constants in (5) and (6) are determined by requiring the adatom concentrations and currents to be equal at the facet boundary. This yields unique solutions for the concentrations, from which the local growth rate, perpendicular to each facet, is calculated as

$$
R_{i}(r)=\frac{d z_{i}}{d t}=\frac{\Omega_{0}}{\tau_{i}} n_{i}(r),
$$

where $\Omega_{0}=a^{3}$ is the atomic volume. The total growth rate is $R_{i}=R_{i}^{(\mathrm{AlAs})}+R_{i}^{(\mathrm{GaAs})}$. The self-limiting width $L_{b}^{*}$ is obtained by requiring that the total growth rate at the boundary between side and bottom facets are equal:

$$
\left.\cos \theta\left[R_{b}^{(\mathrm{AlGaAs})}\right]\right|_{L_{b}=L_{b}^{*}}=\left.\left[R_{s}^{(\mathrm{AlGaAs})}\right]\right|_{L_{b}=L_{b}^{*}} .
$$

There are two types of parameter required for the solution of (8). Those in Table I are geometrical quantities and growth conditions, which are known from experiment. However, the kinetic parameters in Table II, which determine the rates of our surface processes, are difficult to measure directly in an MOVPE reactor and are not readily available from first-principles calculations. Accordingly, we will regard these as fitting parameters, but with restricted values. For example, our experimental observations, combined with studies of molecular-beam epitaxy [29], suggest faster diffusion of Ga on (111)A than on (111)B planes. The differences between atomic Ga and $\mathrm{Al}$ imply a shorter diffusion length and, therefore, a faster incorporation rate for Al. A shorter lifetime on the base facet gives a higher incorporation rate, which accounts for the capillarity-induced enhancement of the atom to be incorporated on that facet. Table II compiles the kinetic parameters for $\mathrm{Al}$ and $\mathrm{Ga}$ on each facet.

Figure 3(a) compares the experimental data collected from batch I and the calculated $L_{b}^{*}$ as a function of alloy composition at $T_{G}=938 \mathrm{~K}$. Apart from the quantitative

TABLE I. Experimental parameters used in (8) to determine the self-limited width in a conical recess [Fig. 2(c)]. $a$ is the thickness of $1 \mathrm{ML}, H$ the depth of the recess, $\theta$ the angle between the bottom and side facets, and $T_{G}$ the growth temperature.

\begin{tabular}{c|c}
\hline \hline Parameter & Value \\
\hline$a$ & $2.71 \times 10^{-10} \mathrm{~m}$ \\
\hline$H$ & $22140 \mathrm{a}=6 \times 10^{-6} \mathrm{~m}$ \\
\hline$\theta$ & $75^{\circ}$ \\
\hline$T_{G}$ & $870-938 \mathrm{~K}$ \\
\hline \hline
\end{tabular}


TABLE II. Kinetic parameters used in (8) to determine the solution of the self-limited width.

\begin{tabular}{c||c|c}
\hline \hline Parameter & $\mathrm{Al}$ & $\mathrm{Ga}$ \\
\hline$E_{b}^{D}(\mathrm{eV})$ & 2.10 & 1.70 \\
\hline$E_{s}^{D}(\mathrm{eV})$ & 1.40 & 1.00 \\
\hline$E_{b}^{\tau}(\mathrm{eV})$ & 0.1511 & 0.0294 \\
\hline$E_{s}^{\tau}(\mathrm{eV})$ & 0.1702 & 0.055 \\
\hline$\nu_{b}\left(\mathrm{~s}^{-1}\right)$ & 33.33 & 3.03 \\
\hline$\nu_{s}\left(\mathrm{~s}^{-1}\right)$ & 3.84 & 0.81 \\
\hline$r^{k}$ & 1.6 & 1.1 \\
\hline \hline
\end{tabular}

agreement between experiments and theory, the qualitative trend confirms the experimental observation of a broadening profile as the Ga concentration increases. Ga adatoms diffuse over longer distances than Al, so a higher Ga concentration can be expected to diffuse from the sidewalls toward the basal plane, whereupon the atoms can be promptly incorporated (the lifetime on the bottom being shorter than on the lateral facets). This leads to an increased growth rate on the bottom facet, which is accompanied by a widening of the self-limiting profile, as shown in the inset of Fig. 3(a). More detailed analysis shows that the self-limited profile results not just from kinetics, but also from a delicate balance between geometry and kinetics (see Supplemental Material for details).

Figure 3(b) shows the spatial dependence of the $\mathrm{Ga}$ concentration profiles for different nominal Ga contents. As expected from experimental observations, our theory exhibits an enrichment of the relative Ga growth rate on the bottom facet, leading to an appreciably higher Ga concentration on the base than on the sidewalls, where the Ga concentration is equal to the nominal value. However, as the nominal Ga concentration increases, the local relative growth rate flattens along the basal facet and, for Ga concentrations $\gtrsim 0.8$, exhibits a shallow double maximum at the boundary between the bottom and sidewalls (barely visible in the figure). This is not necessarily unexpected [30], and a refinement of the adatom kinetic parameters could clarify this behaviour, as it results from the interplay between diffusion and incorporation. This is a matter for future work.

To better visualize the segregation effects, Fig. 3(c) shows the enhancement of the relative Ga growth rate in the middle of the template compared to the nominal growth rate. This figure also shows the fit to experimental data in Ref. [31] for the Ga concentration of a pyramidal structure grown under similar conditions to those employed here, calculated from

$$
\mathrm{Ga}^{\mathrm{eff}}=\frac{k(1-x)}{k(1-x)+x},
$$

for nominal $\mathrm{Al}$ content $x$ with $k=8.6$ a fitting param-
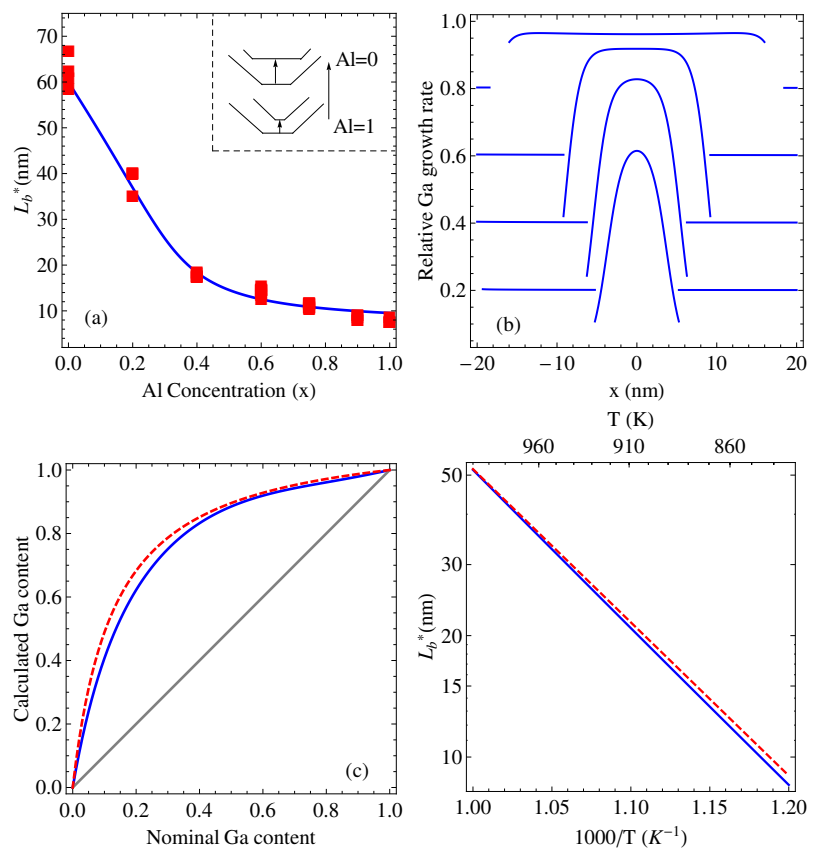

FIG. 3. (Color online) (a) Experimental (red squares) and theoretical (blue curve) values of $L_{b}^{*}$ as a function of $\mathrm{Al}$ content for the pyramidal template at $T_{G}=938 \mathrm{~K}$. The blue curve was calculated from (8) with the parameters in Tables I and II. (b) Calculated steady-state Ga relative growth rates from (7) with the values of $L_{b}^{*}$ obtained from (8). Nominal Ga content is $0.2,0.4,0.6,0.8$ from bottom to top. The discontinuities are caused by differences between kinetic parameters on adjacent facets. (c) Comparison between calculated steady-state (blue, solid trace) and fit to experimental values (red, dashed trace) from Ref. [31] of the Ga content in the middle of the pyramidal recesses as a function of the nominal alloy composition. The straight gray line represents the Ga concentration incorporated with no segregation. (d) Calculated $L_{b}^{*}$ (blue, solid trace) and fit to experimental values (red, dashed trace). $L_{b}^{*}$ is found by solving (8), fixing the $\mathrm{Al}$ content equal to 0.3 and varying $T_{G}$ in the range $830-1000 \mathrm{~K}$.

eter. Good agreement is obtained between this formula and our calculations (the small mismatch might be reduced with further optimization of our kinetic parameters), confirming that our model can predict the actual concentration of $\mathrm{Ga}$ incorporated along the vertical axis of the pyramid, and is revealed, therefore, to be useful for designing structures requiring a specific alloy composition for a particular device application.

To investigate the temperature dependence of the selflimiting width, (8) was solved for $T=830-1000 \mathrm{~K}$ with a fixed $\mathrm{Al}$ concentration of 0.3 . Figure $3(\mathrm{~d})$ compares the calculated values of $L_{b}^{*}$ with the experimental values extrapolated from cross-sectional AFM profiling measurements from the batch II. The model predicts, in nearperfect agreement with the experiments (parameter optimizations again might lead to a better agreement), a widening of the self-limiting width as the temperature increases, owing to the enhanced growth rate on the bot- 
tom of the template as a consequence of capillarity-driven surface diffusion (which increases with the temperature) from the sidewalls toward the basal plane.

In summary, we have introduced a theoretical model which comprehensively reproduces the main experimentally observable phenomena during the growth by MOVPE of pyramidal QDs and VQWRs. The reactiondiffusion equations formulated here account for the interplay between precursor decomposition, adatom diffusion and incorporation on the different crystallographic facets of the seeding template, and can be extended to study the morphological evolution of any patterned surface. These results pave the way toward a reproducible on-demand design of seeded low-dimensional nanostructures and establish solid foundation for the future development of quantum-based technologies. Indeed, our improved understanding of the growth process has enabled us to obtain, for the first time, ordered arrays of entangled photon emitters, rather than isolated emitters, on a single wafer [32].

This research was enabled by the Irish Higher Education Authority Program for Research in Third Level Institutions (2007 to 2011) via the INSPIRE programme, and by Science Foundation Ireland under grants 05/IN.1/I25 and 10/IN.1/I3000. We are grateful to K. Thomas for his support with the MOVPE system.

[1] M. Ozdemir and A. Zangwill, J. Vac. Sci. Technol. A 10, 684 (1992).

[2] E. Kapon, D. M. Hwang, and R. Bhat, Phys. Rev. Lett. 63, 430 (1989).

[3] K. Dzurko, E. Menu, C. Beyler, J. Osinski, and P. Dapkus, Appl. Phys. Lett. 54, 105 (1989).

[4] A. Madhukar, K. C. Rajkumar, and P. Chen, Appl. Phys. Lett. 62, 1547 (1993).

[5] S. Koshiba, H. Noge, H. Akiyama, T. Inoshita, Y. Nakamura, A. Shimizu, Y. Nagamune, M.Tsuchiya, H. Kano, H. Sakaki, and K. Wada, Appl. Phys. Lett. 64, 363 (1994).

[6] P. Atkinson, M. Ward, S. Bremner, D. Anderson, T. Farrow, G. Jones, A. Shields, and D. Ritchie, Physica E 32, 21 (2006).

[7] J. Skiba-Szymanska, A. Jamil, I. Farrer, M. B. Ward, C. A. Nicoll, D. J. P. Ellis, J. P. Griffiths, D. Anderson, G. A. C. Jones, D. A. Ritchie, A. J. Shields, Nanotechnology 22, 065302 (2011).

[8] S. Kiravittaya, A. Rastelli, and O. G. Schmidt, Appl. Phys. Lett. 88, 043112 (2006).

[9] A. Hartmann, L. Loubies, F. Reinhardt, A. Gustafsson, A. Sadeghi, and E. Kapon, Appl. Surf. Sci. 123/124, 329 (1998).

[10] E. Kapon, G. Biasiol, D.M. Hwang, and E. Colas, Microelec Jour 26, 881-886 (1995).

[11] M. H. Baier, S. Watanabe, E. Pelucchi, and E. Kapon, Appl. Phys. Lett. 84, 648 (2004).

[12] L. O. Mereni, V. Dimastrodonato, and R. J. Young, E. Pelucchi, Appl. Phys. Lett. 94, 223121 (2009).
[13] A. Mohan, P. Gallo, M. Felici, B. Dwir, A. Rudra, J. Faist, and E. Kapon, Small 6, 1268 (2010).

[14] P. Gallo, M. Felici, B. Dwir, K. A. Atlasov, K. F. Karlsson, A. Rudra, A. Mohan, G. Biasiol, L. Sorba, and E. Kapon, Appl. Phys. Lett. 92, 263101 (2008).

[15] A. Mohan, M. Felici, P. Gallo, B. Dwir, A. Rudra, J. Faist, and E. Kapon, Nat. Photonics 4, 302 (2010).

[16] G. Bester, S. Nair, and A. Zunger, Phys. Rev. B 67, 161306 (2003).

[17] M. A. Dupertuis, K. F. Karlsson, D. Y. Oberli, E. Pelucchi, A. Rudra, P. O. Holtz, and E. Kapon, Phys. Rev. Lett. 107, 127403 (2011).

[18] A. L.-S. Chua, E. Pelucchi, A. Rudra, B. Dwir, E. Kapon, A. Zangwill, and D. D. Vvedensky, Appl. Phys. Lett. 92, 013117 (2008).

[19] A. Dalla Volta, D. D. Vvedensky, N. Gogneau, E. Pelucchi, A. Rudra, B. Dwir, E. Kapon, and C. Ratsch, App. Phys. Lett. 88, 203104 (2006).

[20] All samples were grown in a low-pressure horizontal reactor on substrates patterned by deep UV photolithography and wet chemical etching. The structure comprises a GaAs buffer and pairs of $120 \mathrm{~nm} \mathrm{Al}_{x} \mathrm{Ga}_{1-x}$ As and 15/25 $\mathrm{nm}$ GaAs markers grown at $0.5 \mathrm{ML} / \mathrm{s}$ with a $\mathrm{V} / \mathrm{III}$ ratio $\sim 750$.

[21] The AFM scans were acquired under atmospheric conditions immediately after cleaving the samples. Only samples for which the cleaving direction crosses the template in the proximity of the center, corresponding to the base of the profile, were selected. To avoid tip-resolutionrelated problems, tip shape characterization and image decorrelation processing were also carried out.

[22] E. Pelucchi, V. Dimastrodonato, A. Rudra, K. Leifer, E. Kapon, L. Bethke, P. A. Zestanakis, and D. D. Vvedensky, Phys. Rev. B 83, 205409 (2011).

[23] I. Horcas, R. Fernandez, J. M. Gomez-Rodriguez, J. Colchero, J. Gomez-Herrero, and A. M. Baro, Rev. Sci. Instrum. 78, 013705 (2007).

[24] P. Chen, J. Y. Kim, A. Madhukar, and N. M. Cho, J. Vac. Sci. Technol. B 4, 890 (1986).

[25] T. Shitara, D. D. Vvedensky, M. R. Wilby, J. Zhang, J. H. Neave, and B. Joyce, Phys. Rev. B 46, 6815 (1992).

[26] E. Pelucchi, S. Watanabe, K. Leifer, Z. Zhu, B. Dwir, P. De Los Rios, and E. Kapon, Nano Lett. 7, 1282 (2007).

[27] A. Pimpinelli and J Villain, Physics of Crystal Growth (Cambridge University Press, Cambridge, UK, 1998).

[28] J. Gravesen, M. Willatzen, and L. C. Lew Yan Voon, J. Math. Phys. 46, 012107 (2005).

[29] T. Takebe, M. Fujii, T. Yamamoto, K. Fujiita, and T. Watanabe, J. Appl. Phys. 81, 7273 (1997).

[30] G. Biasiol, Ph.D. Thesis no 1859, Formation Mechanisms of Low-Dimensional Semiconductor Nanostructures Grown by OMCVD on Nonplanar Substrates (Swiss Federal Institute of Technology, Lausanne, 1998).

[31] Q. Zhu, E. Pelucchi, S. Dalessi, K. Leifer, M.-A. Dupertuis, and E Kapon, Nano Lett. 6, 1036 (2006).

[32] G. Juska, V. Dimastrodonato, L. O. Mereni, A. Gocalinska, and E. Pelucchi, unpublished.

\section{SUPPLEMENTAL MATERIAL}

The calculated self-limited width for several angles between the basal and lateral facets has been calculated, 
keeping all the other parameters in (8) fixed. A pronounced decrease of the profile is seen for increasing angle values, due to an increased growth rate on the lateral (111)A with respect to the (111)B bottom facets.

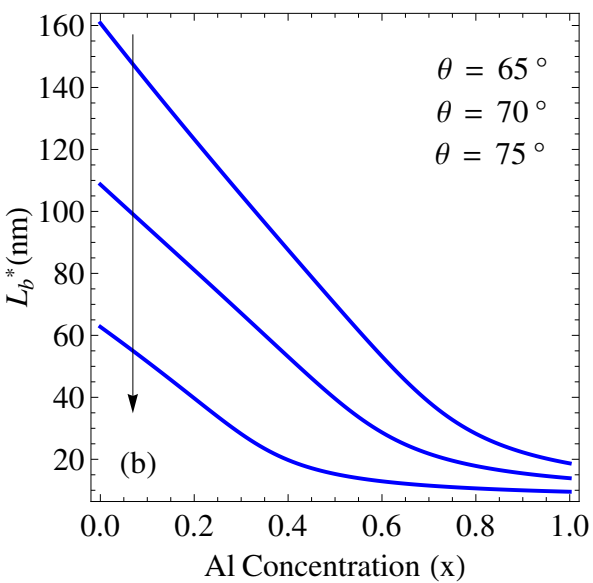

FIG. 4. Calculated self-limiting width as a function of $\mathrm{Al}$ concentration obtained from (8) for different values of the basal angle $\theta$ [Fig. 2 (c)]. As the profile becomes sharper (greater angles), the width decreases. 